\title{
Effect of Non-Uniform Heat Generation on Unsteady MHD Non-Darcian Flow over a Vertical Stretching Surface with Variable Properties
}

\author{
M. Muthtamilselvan ${ }^{1 \dagger}$, D. Prakash ${ }^{1}$ and D. H. Doh ${ }^{2}$ \\ ${ }^{1}$ Department of Applied Mathematics, Bharathiar University, Coimbatore-641 046, India \\ ${ }^{2}$ Division of Mechanical and Energy Systems Engineering, College of Engineering, Korea Maritime \\ University, Busan 606-791, South Korea \\ $\uparrow$ Corresponding Author Email: muthtamill@yahoo.co.in
}

(Received January 10, 2013; accepted April 3, 2013)

\begin{abstract}
The effect of non-uniform heat generation on an unsteady MHD laminar boundary layer flow of viscous, incompressible fluid over a vertical stretching plate embedded in a sparsely packed porous medium is investigated numerically. The flow in the porous medium is governed by Brinkman-Forchheimer extended Darcy model. The variation of porosity, permeability and thermal conductivity is assumed. By applying similarity analysis, the governing partial differential equations are transformed into a set of time dependent non-linear coupled ordinary differential equations and they are solved by Runge-Kutta Fehlberg Method along with shooting technique. The effects of governing parameters on the dimensionless velocity and temperature distributions for uniform permeability (UP) and variable permeability (VP) of the porous medium are discussed graphically. Also, the local skin friction coefficient and the rate of heat transfer are computed for various pertinent parameters governing the problem. Moreover, the numerical results obtained in this study is compared with the existing literature and found they are in good agreement.
\end{abstract}

Keywords: MHD, Brinkman-Forchheimer model, Stretching plate, Non-uniform heat source/sink, Variable porosity and permeability, Variable thermal conductivity.

\section{NOMENClATURE}

\begin{tabular}{|c|c|c|}
\hline \multicolumn{2}{|c|}{$a, b, c, d, d^{*}$ constants } & $T \& t$ fluid temperature and time \\
\hline & unsteady parameter & given temperature at the sheet \\
\hline $\begin{array}{l}A^{*}, B^{*} \\
B_{0}\end{array}$ & $\begin{array}{l}\text { dimensionless heat generation parameters } \\
\text { uniform magnetic field }\end{array}$ & $\begin{array}{l}\text { constant temperature of the fluid far away } \\
\text { from the sheet }\end{array}$ \\
\hline$C_{b}$ & empirical constant of second order & axial and normal co-ordinate \\
\hline$C_{f}$ & skin-friction & velocities in $\mathrm{x}, \mathrm{y}$ directions respectively \\
\hline $\begin{array}{l}c_{p} \\
f\end{array}$ & $\begin{array}{l}\text { specific heat at constant pressure } \\
\text { dimensionless stream function }\end{array}$ & velocity of the stretching surface \\
\hline$g$ & acceleration due to gravity & Greek symbols \\
\hline$G r$ & local Grashof number & \\
\hline$k(y)$ & permeability of the porous medium & $\alpha_{\infty}, \alpha^{*}$ thermal diffusivity and ratio of viscosities \\
\hline $\begin{array}{l}k_{0} \\
\text { the ed }\end{array}$ & $\begin{array}{l}\text { permeability of the porous medium at } \\
\text { ge of the boundary laver }\end{array}$ & porosity of the porous medium \\
\hline$K(T)$ & thermal conductivity & kinematic viscosity \\
\hline$K_{\infty}$ & $\begin{array}{l}\text { thermal conductivity far away from the } \\
\text { sheet }\end{array}$ & $\begin{array}{l}\text { inertial parameter, thermal expansion co- } \\
\text { eff. }\end{array}$ \\
\hline$M$ & magnetic parameter & dynamic viscosity \\
\hline$N u_{x}$ & Nusselt number & stream function \\
\hline $\operatorname{Pr}$ & Prandtl number & density \\
\hline $\operatorname{Re}$ & local Reynolds number & permeability parameter \\
\hline $\begin{array}{l}q_{w} \\
Q^{*}\end{array}$ & $\begin{array}{l}\text { local heat flux at the sheet } \\
\text { volumetric non-uniform heat generation }\end{array}$ & $\begin{array}{l}\text { shear stress } \\
\text { dimensionless temperature variable } \\
\text { buoyancy parameter }\end{array}$ \\
\hline
\end{tabular}




\section{INTRODUCTION}

The study of MHD flow and heat transfer over a stretching surface has gained considerable interest because of its extensive engineering applications, such as in the extrusion of a polymer sheet from a die, glass fiber and paper production. These flows occur in many manufacturing processes in modern industry, such as hot rolling, hot extrusion, wire drawing and continuous casting. Considering of its importance, those flow have been studied by several research groups (Sakiadis 1961; Sparrow and Cess 1961; Sing and Cowling 1963; Riley 1964). The continuing interest in heat transfer and fluid flow through porous media is mainly due to several engineering and geophysical fields such as cooling of nuclear reactors, enhanced oil recovery, thermal insulation drying of porous solids, solid matrix heat exchanges, geothermal and petroleum resources, ceramic processing, filtration processes, chromatography, etc.

The problem of mixed convection flow past a stretching sheet embedded in porous medium arise in some metallurgical processes which involve the cooling of continuous strips or filaments by drawing them through quiescent fluid and the rate of cooling can be better controlled and final product of desired characteristics can be achieved if the strips are drawn through porous media ( $\mathrm{Pal}$ and Mondal 2011). A comprehensive review of convection through porous medium was reported by Nield and Bejan (1992) and by Ingham and Pop (1998). Lai and Kulacki (1991) have studied the boundary layer mixed convection flow of heat and mass transfer over a vertical plate embedded in a saturated porous medium with constant wall temperature and constant heat flux. Recently, Makinde and Aziz (2010) investigated the mixed convection flow from a vertical plate embedded in a porous medium with magnetic effect and convective boundary condition.

One common feature of all the above-mentioned fluid saturated porous media studies deal with the Darcy flow model. But in many practical applications, for example packed sphere beds, the porous medium is bounded by an impermeable wall has higher flow rates, and reveals nonhomogeneous porosity variation near the wall, making Darcy's law inapplicable. Such a case, the boundary and inertial effects should be included. The boundary and inertia effects on forced convective heat transfer from a flat plate were first examined by Vafai and Tien (1981). These effects were shown to decrease the velocity in the thermal boundary layer and reduce the heat transfer rate. Recently, Pal and Mondal (2011) studied the effect of radiation on the heat and mass transfer past a stretching sheet in a non-darcian porous medium. They found that the radiation parameter enhances the skin-friction coefficient and sherwood number whereas the local nusselt number decreases.
Owing to adding time effect, the transient heat transfer is usually difficult to solve with either an analytical approach or numerical method. Examples of transient convective flows are numerous such as cooling of electronic devices in which the heat generation is not constant but time varying. First study on transient boundary layer on flat plate was made by Johnson and Cheng (1978). Raptis (1983) studied the case of two-dimensional free convection over a vertical plate embedded in a porous medium using the perturbation method. Al-Nimr and Masoud (1998) analyzed the problem of transient free convection flow over an impermeable vertical flat plate embedded in porous medium using Laplace transformation method. Anand Rao et al. (2012) investigated the effect of chemical reaction on unsteady MHD free convection flow over a semi-infinite vertical porous plate. Recently, Reddy et al. (2013) found the exact solutions of the unsteady MHD flow, heat and mass transfer over a moving vertical porous plate using Laplace transform technique.

The study of heat generation or absorption effects in moving fluid is important in view of several physical problems. Due to the fast growth of electronic technology, effective cooling of electronic equipment has become warranted and cooling of electronic equipment ranges from individual transistors to main frame computers and from energy suppliers to telephone switch boards. Several authors have investigated the heat transfer problems by considering temperature dependent heat source/sink (Chamkha and Ahmed 2011; Anand Rao 2012 ; Prakash et al. 2012, etc). Although, exact modelling of non-uniform heat generation is quite difficult, some simple mathematical model can express its average behavior for most physical situations. For example, in many situations there may be appreciable temperature difference between the surface and the ambient fluid. In such a case, non-uniform heat generation plays a crucial role and exerts strong influence on the heat transfer characteristics. AboEldahab and El-Aziz (2004) studied the problem to involve a space-dependent exponentially decaying with internal heat generation or absorption. Abel et al. (2007) and Bataller (2007) investigated the effects of non-uniform heat source on visco-elastic fluid flow and heat transfer over a stretching sheet.

In most of the papers, the thermo physical properties of the ambient fluid were assumed to be constant. Based on previous investigations (Chiam 1996,1998 ; Mahmoud 2007 ; Rahman et al.2009; Sharma and Singh 2009 ; Mahanti and Gaur 2009 ; Prasad et al. 2013), it is well known that these physical properties may change with temperature, especially thermal conductivity. For lubricating fluids, heat generated by internal friction and the corresponding rise in the temperature affects the physical properties of the fluid and so the properties of the fluid are no longer assumed to be constant. 
The increase in temperature leads to increase in the transport phenomena by reducing the physical properties across the thermal boundary layer and the heat transfer at the wall is also affected. Therefore to predict the flow and heat transfer rates, it is necessary to take into account the variable fluid properties.

The present study is to investigate the non-uniform heat generation effect on unsteady MHD boundary layer flow of an incompressible viscous fluid and heat transfer over a vertical surface embedded in the porous medium with variable porosity and variable thermal conductivity. By using similarity approach, the transport equations are transformed into non-linear ordinary differential equations and they are solved by Runge-Kutta-Fehlberg with shooting method. The present results are compared with previously obtained solutions and they are in good agreement. The behavior of the velocity, temperature, skin-friction and heat transfer has been discussed for a range of physical parameters.

\section{MATHEMATICAL FORMULATION}

Consider an unsteady two-dimensional laminar boundary layer flow over a continuous moving stretching plate in a viscous incompressible electrically conducting fluid saturated porous medium of variable porosity, permeability and thermal conductivity. A uniform magnetic field $B_{0}(t)=\hat{B}_{0}(1-c t)^{-1 / 2}$ is applied in the direction perpendicular to the stretching surface. Since the transverse applied magnetic field and magnetic Reynolds number are assumed to be small, the induced magnetic field can be neglected. The $x$-axis is taken along the stretching plate in the direction of the motion and the $y$-axis is perpendicular to the plate in the outward direction towards the fluid of ambient temperature $T_{\infty}$ (See Fig. 1).

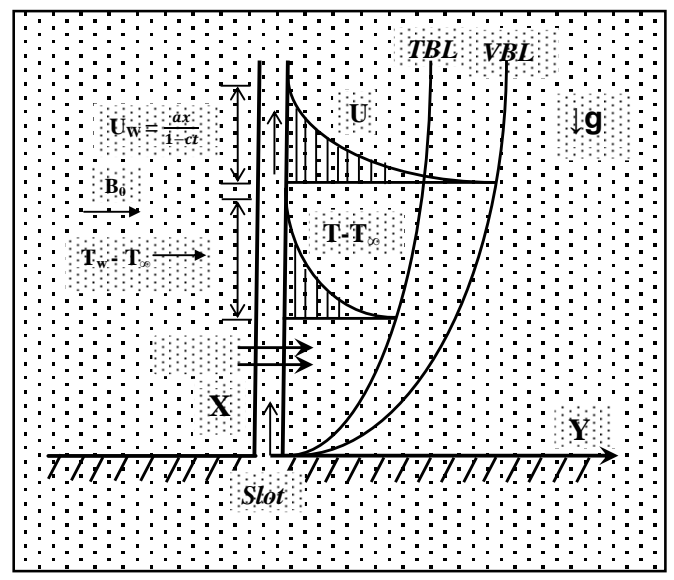

Fig. 1 Physical configuration \& coordinate system

We assume that for time $t<0$ the fluid and heat flows are steady. The unsteady fluid and heat flows start at $t=0$, the plate is being stretched with the velocity $U_{w}(x, t)=a x /(1-c t)$ along the $x$ axis, where $a$ (stretching rate) and $c$ are positive constants having dimension $t^{-1}$ (with $c t<1, c \geq 0$ ). The surface temperature of the plate varies with the distance $x$ from the slot and time $t$ in the form $T_{w}=T_{\infty}+(b x / 1-c t)$ where $b$ is a constant and has dimension temperature/length.

The porous medium is isotropic and homogeneous. Local thermal equilibrium is assumed. With the inclusion of quadratic drag, inertial and boundary effects, the governing boundary layer equations for unsteady two dimensional flow can be written in the following form:

$$
\begin{aligned}
& \frac{\partial u}{\partial x}+\frac{\partial v}{\partial y}=0 \\
& \frac{\partial u}{\partial t}+u \frac{\partial u}{\partial x}+v \frac{\partial u}{\partial y}=-\frac{\mu \varepsilon(y)}{\rho k(y)} u+\frac{\bar{\mu}}{\rho} \frac{\partial^{2} u}{\partial y^{2}} \\
& -\frac{C_{b} \varepsilon^{2}(y)}{\sqrt{k(y)}} u^{2}+g \beta\left(T-T_{\infty}\right)-\frac{\sigma_{m} B_{0}^{2}(t)}{\rho} u \\
& \rho c_{p}\left(\frac{\partial T}{\partial t}+u \frac{\partial T}{\partial x}+v \frac{\partial T}{\partial y}\right)=\frac{\partial}{\partial y}\left(K(T) \frac{\partial T}{\partial y}\right)+Q^{*}
\end{aligned}
$$

The initial and boundary conditions are:

$$
\begin{aligned}
& u=U_{w}(x, t), v=0, T=T_{w}(x, t) \text { at } y=0 \\
& u \rightarrow 0, T \rightarrow T_{\infty} \text { as } y \rightarrow \infty
\end{aligned}
$$

where $u$ and $v$ are the velocity components along $x$ and $y$ directions, respectively. $T$ is the temperature of the fluid, $\rho$ is the fluid density, $\bar{\mu}$ is the effective viscosity of the fluid, $\mu$ is the fluid viscosity, $k(y)$ is the variable permeability of the porous medium, $\varepsilon(y)$ is the porosity of the saturated porous medium, $C_{b}$ is the empirical constant of the second-order resistance term due to inertia effect, $g$ is the acceleration due to gravity, $\beta$ is the coefficient of thermal expansion, $c_{p}$ is the specific heat at constant pressure, $\sigma_{\mathrm{m}}$ is the magnetic permeability of the fluid and $\mathrm{B}_{0}(\mathrm{t})$ is the applied magnetic field.

Savvas et al. (1994) observed that for liquid metals, the thermal conductivity varies linearly with temperature in the range 0 to $400^{\circ} \mathrm{C}$. Following Chiam (1996, 1998) and Savvas et al. (1994), we consider the specific model for variable thermal conductivity as

$$
K(T)=K_{\infty}\left(1+\varepsilon \frac{T-T_{\infty}}{T_{w}-T_{\infty}}\right)
$$

where $\mathrm{K}_{\infty}$ is the thermal conductivity of the fluid far away from the stretching surface, $\varepsilon$ is a small parameter known as the variable thermal conductivity parameter.

The term $Q^{*}$ in the right hand side of Eq.(2.3) is due to non-uniform heat generation which is defined (Abo-Eldahab and El-Aziz 2004; Pal 2011; Das 2012) as

$$
Q^{*}=\frac{K_{\infty} U_{w}(x, t)}{x v}\left[A^{*}\left(T_{w}-T_{\infty}\right) e^{-\eta}+B^{*}\left(T-T_{\infty}\right)\right]
$$

where $\mathrm{A}^{*}$ and $\mathrm{B}^{*}$ are the coefficient of exponentially decaying space and temperature- 
dependent heat source/ sink, respectively. It is to be noted that the case $A^{*}>0, \quad B^{*}>0$ corresponds to internal heat generation and that $A^{*}<0, B^{*}<0$, corresponds to internal heat absorption.

We now introduce a stream function $\psi(x, y, t)$, which is defined by

$u=\frac{\partial \psi}{\partial y}, \quad v=-\frac{\partial \psi}{\partial x}$

The mathematical analysis of the problem is simplified by introducing the dimensionless functions $f, \theta$ in terms of the similarity variable $\eta$ [see Ishak et al. (2009a), Prakash et al. (2014)] as

$$
\eta=y \sqrt{\frac{U_{w}}{v x}}, \quad \psi=\sqrt{v x U_{w}} f(\eta), \quad \theta=\frac{T-T_{\infty}}{T_{w}-T_{\infty}}
$$

We consider the variable permeability $k(\eta)$ and variable porosity $\varepsilon(\eta)$ are to decrease exponentially with the normal distance to the wall, from a value close to one at the solid boundaries to $\mathrm{k}_{0}$ and $\varepsilon_{0}$ value at the edge of the boundary layer (see Chandrasekhara and Namboodiri 1985).

$$
\begin{aligned}
& k(\eta)=k_{0}\left(1+d e^{-\eta}\right) \\
& \varepsilon(\eta)=\varepsilon_{0}\left(1+d^{*} e^{-\eta}\right)
\end{aligned}
$$

where $\mathrm{k}_{0}$ and $\varepsilon_{0}$ are the permeability and porosity at the edge of the boundary layer respectively. For variable permeability, $d$ and $\mathrm{d}^{*}$ are treated as constants having values 3.0 and 1.5 respectively and for uniform permeability $d=d^{*}=0$.

Substituting Eqs.(2.6) to (2.10) into Eqs.(2.1) to (2.5), we get the following transformed equations:

$f^{\prime \prime \prime}+f f^{\prime \prime}-f^{\prime 2}-A\left(f^{\prime}+\frac{1}{2} \eta f^{\prime \prime}\right)+\lambda \theta-$

$\frac{\alpha^{*}}{\sigma \operatorname{Re}}\left(\frac{1+d^{*} e^{-\eta}}{1+d e^{-\eta}}\right) f^{\prime}-\beta^{*} \frac{\left(1+d^{*} e^{-\eta}\right)^{2}}{\left(1+d e^{-\eta}\right)^{1 / 2}} f^{\prime 2}-M f^{\prime}=0$

$\left((1+\varepsilon \theta) \theta^{\prime}\right)^{\prime}-\operatorname{Pr}\left(f^{\prime} \theta-f \theta^{\prime}\right)-\operatorname{Pr} A\left(\theta+\frac{1}{2} \eta \theta^{\prime}\right)$

$+\left(A^{*} e^{-\eta}+B^{*} \theta\right)=0$

and the boundary conditions

$$
\begin{aligned}
& f(0)=0, \quad f^{\prime}(0)=1, \quad \theta(0)=1 \\
& f^{\prime}(\infty)=0, \quad \theta(\infty)=0
\end{aligned}
$$

where prime denotes the differentiation with respect to $\eta, A=c / a$ is the unsteady parameter, $M=\frac{\sigma_{m} \hat{B}_{0}^{2}}{\rho a}$ is the local magnetic parameter, ${ }^{\beta^{*}}=\frac{C_{L} \varepsilon_{0}^{2} x}{\sqrt{k_{0}}}$ is the local inertial parameter, $\lambda=\mathrm{Gr}_{\mathrm{Re}}^{2}$ is the free convection parameter, $\operatorname{Pr}=v / \alpha_{\infty}$ is the Prandtl number, where $v=\bar{\mu} / \rho$ and $\alpha_{\infty}=K_{\infty} / \rho c_{p}, \quad G r=\frac{g \beta\left(T_{w}-T_{\infty}\right) x^{3}}{v^{2}}$ is the local Grashof number, $\operatorname{Re}=U_{w} x / v$ is the local Reynolds number, $\alpha^{*}=\mu / \bar{\mu}$ is the ratio of viscosities and $\sigma=k_{0} / x^{2} \varepsilon_{0}$ is the local permeability parameter.
The important characteristics of the flow are the skin-friction co-efficient $\mathrm{C}_{\mathrm{f}}$ and the local Nusselt number $\mathrm{Nu}_{\mathrm{x}}$, which are defined as

$$
C_{f}=\frac{\tau_{w}}{\rho U_{w}^{2} / 2}, N u_{x}=\frac{x q_{w}}{K_{\infty}\left(T_{w}-T_{\infty}\right)}
$$

where the wall shear stress $\tau_{\mathrm{w}}$ and the surface heat flux $\mathrm{q}_{\mathrm{w}}$ are given by

$$
\tau_{w}=\mu\left(\frac{\partial u}{\partial y}\right)_{y=0}, \quad q_{w}=-K_{\infty}\left(\frac{\partial T}{\partial y}\right)_{y=0}
$$

Substituting Eq.(2.16) in Eq.(2.15), we get

$$
\frac{1}{2} C_{f} \sqrt{\operatorname{Re}_{x}}=f^{\prime \prime}(0), \quad N u_{x} / \sqrt{\operatorname{Re}_{x}}=-\theta^{\prime}(0)
$$

where $\operatorname{Re}_{x}=U_{w} x / v$ is the local Reynolds number which is based on the surface velocity.

\section{Numerical Procedure}

The coupled system of equations Eq. (2.11) to (2.12) is highly non-linear. Most of the physical systems are inherently non-linear in nature and are of great interest to physicists, engineers and mathematicians. Problems involving non-linear ordinary differential equations are difficult to solve exactly. So, the governing equations together with the boundary conditions have to be solved numerically.

The system of equations subject to the boundary conditions (2.13)-(2.14) was solved numerically by Runge-Kutta-Fehlberg method along with shooting technique using MATLAB. Its accuracy and robustness has been repeatedly confirmed in various heat transfer papers. The asymptotic boundary conditions given by Eq.(2.14) were replaced by using a value of 6 for the similarity variable $\eta_{\max }$ as follows:

$$
\eta_{\max }=6, f^{\prime}(6)=0, \theta(6)=0
$$

Table 1 Comparison of results of the wall temperature gradient with Ishak et al. (2009b) and Vajravelu et al. (2013) for

$\left(M=\beta^{*}=A=0, \frac{\alpha^{*}}{\sigma \operatorname{Re}}=0, A^{*}=B^{*}=0\right)$

\begin{tabular}{|c|c|c|c|c|c|}
\hline$\varepsilon$ & $\lambda$ & $P r$ & $\begin{array}{c}\text { Ishak } \\
\text { et al. }\end{array}$ & $\begin{array}{c}\text { Vajravelu } \\
\text { et al. }\end{array}$ & $\begin{array}{c}\text { Present } \\
\text { results }\end{array}$ \\
\hline 0 & 0 & 0.72 & 0.8086 & 0.808836 & 0.80883589 \\
& & 1.0 & 1.0000 & 1.000000 & 1.00000008 \\
& & 3.0 & 1.9237 & 1.923687 & 1.92365749 \\
& & 10.0 & 3.7207 & 3.720788 & 3.72064164 \\
\hline 0 & 1.0 & 1.0 & 1.0873 & 1.087206 & 1.08726848 \\
& 2.0 & & 1.1429 & 1.142298 & 1.14233042 \\
& 3.0 & & 1.1853 & 1.185197 & 1.18528379 \\
\hline 0.1 & 1.0 & 1.0 & - & 1.018446 & 1.01861813 \\
\hline
\end{tabular}

Pantokratoras (2009) noticed that the erroneous result is found by many researchers in the field of 
convective heat and mass transfer because of taking small far field asymptotic value of $\eta_{\max }$ during their numerical computation. Here, the choice of $\eta_{\max }=6$ ensured that all numerical solutions approached the asymptotic values correctly.

In order to check the accuracy of the numerical solution procedure used, a comparison of wall temperature gradient $-\theta^{\prime}(0)$ for various values of $\mathrm{Pr}$, $\varepsilon, \lambda$ with those of Ishak et al. (2009b) and Vajravelu et al. (2013) under certain limiting conditions is shown in Table 1. From the table, the present results are found to be in good agreement.

\section{ReSUlts AND Discussion}

In order to understand the physical nature of the problem and the effects of various parameters like Magnetic number $M$, free convection parameter $\lambda$, variable thermal conductivity parameter $\varepsilon$, the unsteadiness parameter A, the Prandtl number Pr, the space and time dependent heat generation parameters $\boldsymbol{A}^{* *}, \boldsymbol{B}^{* \prime}$, we have computed the numerical solutions of the velocity and temperature profiles.

The influence of the magnetic parameter $M$ on the velocity and temperature profiles with fixed values of other parameters for both steady and unsteady cases are depicted in Fig.2 and Fig.3. It can be seen that with the fixed value of unsteady parameter, the effect of increasing of magnetic parameter is to decrease the velocity profile near the plate $(0 \leq \eta \leq 6)$. This is due to the fact that the transverse magnetic field gives rise to a resistive-type of force called the Lorentz force. This force has a tendency to slow down the motion of the fluid which results in reducing the velocity profiles. Further, the temperature profile increases with the increasing of magnetic parameter, due to interaction of applied magnetic field and fluid particles. These results qualitatively agree with the expectations. Moreover, with the fixed values of magnetic parameter, the effect of increasing values of unsteady parameter $A$ is to decrease the velocity and temperature field and hence it reduce the momentum and thermal boundary layer thickness.

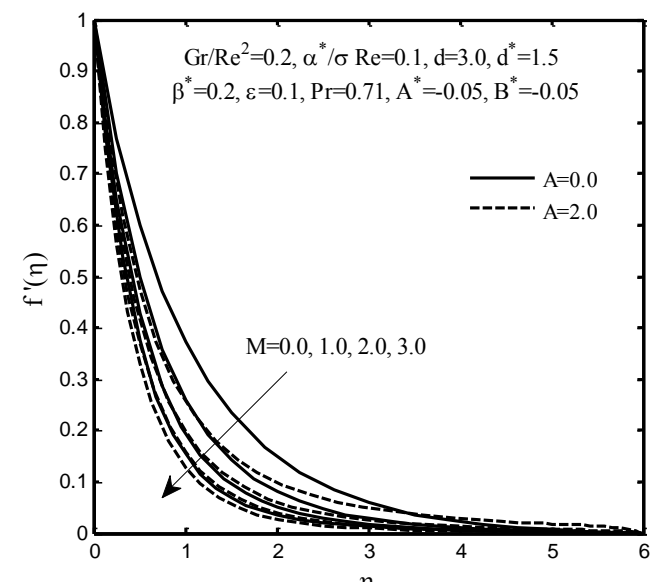

Fig. 2 Velocity profile for different values of magnetic and unsteady parameters

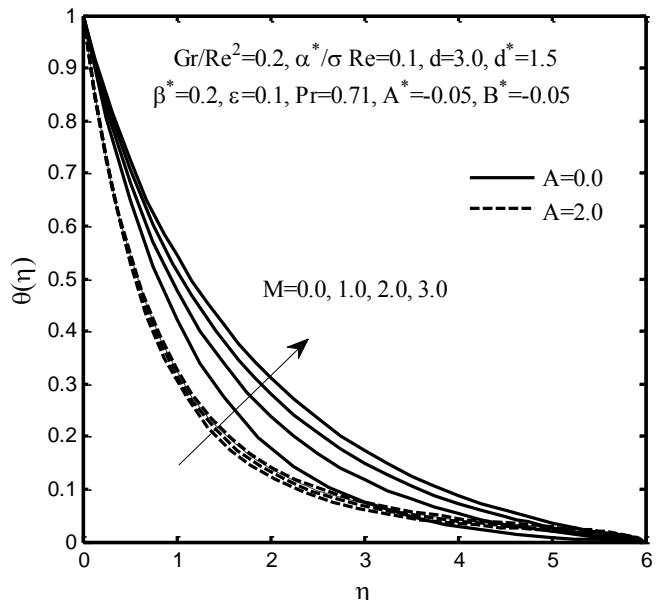

Fig. 3 Temperature profile for different values of magnetic and unsteady parameters

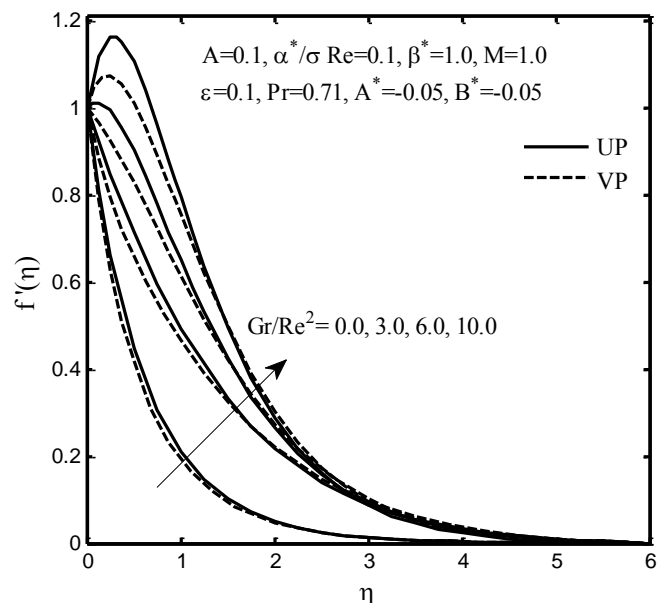

Fig. 4 Velocity profile for different values of buoyancy parameter

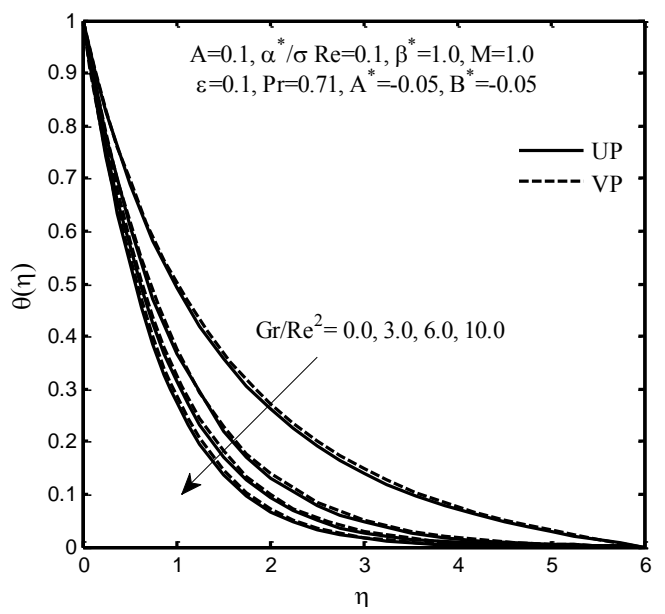

Fig. 5 Temperature profile for different values of buoyancy parameter

The effect of free convection parameter $\lambda=\mathrm{Gr} / \mathrm{Re}^{2}$ for the cases of UP and VP on velocity and temperature fields are shown in Fig.4 and Fig.5. It can be observed that an increase in the buoyancy parameter is to increase the velocity field and also increase the boundary layer thickness. Also in the 
case of higher buoyancy parameter, a peak is observed near the stretching boundary which exponential decreases away from the stretching boundary layer. This means that the ambient fluid velocity near the surface is higher than the stretching surface velocity. Whereas in temperature field, an increase in $\lambda$ is to decrease in thermal boundary layer thickness. Moreover, when comparing the velocity and temperature profile for variable permeability (VP) with uniform permeability (UP) for a fixed value of $\lambda$, there is a decrease in momentum boundary layer thickness and increase in thermal boundary layer thickness.

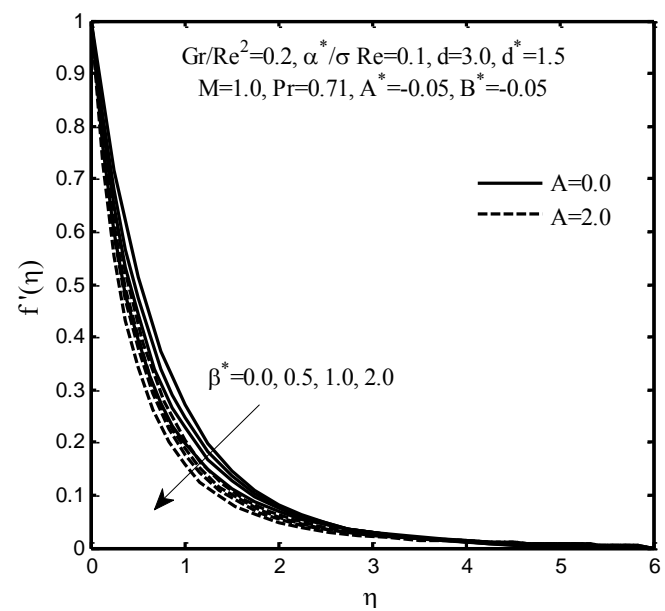

Fig. 6 Velocity profile for different values of inertial parameter and unsteady parameter

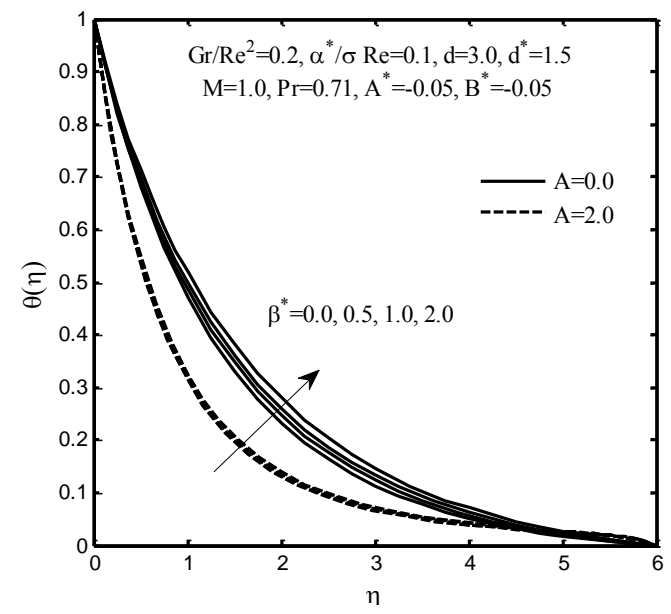

Fig. 7 Temperature profile for different values of inertial parameter and unsteady parameter

Figures 6 and 7 show the effect of inertial parameter on the velocity and temperature profiles for both steady and unsteady cases. It is observed that the inertia effect tends to decrease the velocity, since the fluid inertia provides an additional pressure loss in the flow field for both steady and unsteady cases. Moreover, the temperature increases as the inertial parameter increases due to the fact that the flow inertia force retards the momentum transport. This, in turn produces decrease in the velocity and increase in the fluid temperature. In addition, a slight increase in the thermal boundary layer thickness is observed as a result of increasing the inertial parameter for unsteady case comparing with steady one.

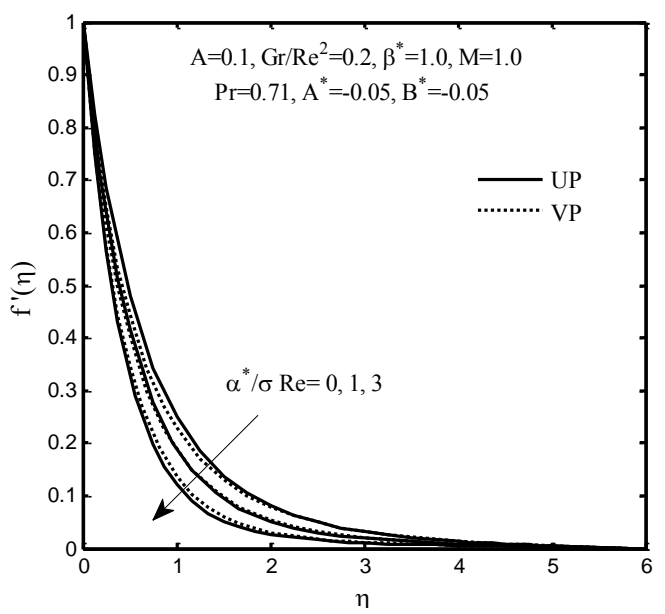

Fig. 8 Velocity profile for different values of $\alpha$ / $\sigma \mathrm{Re}$

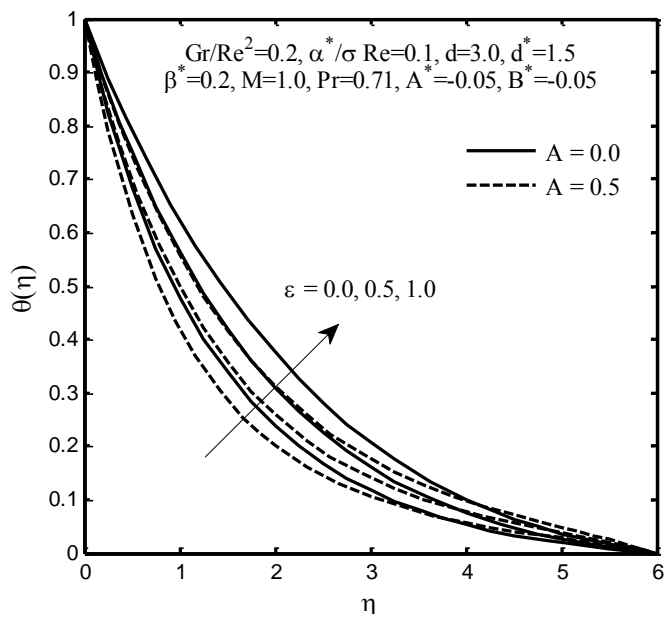

Fig. 9 Temperature profile for different values of

The influence of $\alpha^{*} / \sigma \operatorname{Re}$ with UP and VP on the velocity profile is shown in Fig.8. It is observed that in the presence of porous medium causes higher retardation to the fluid which reduces the velocity and momentum boundary layer thickness. It is noted that, there is a significant increase in VP than UP for the higher $\alpha^{\prime \prime} / \sigma$ Re . Fig. 9 is plotted to demonstrate the temperature profile for the selected values of thermal conductivity parameter $\varepsilon$ and unsteady parameter $A$ with the fixed values of other parameters. It can be seen that an increasing $\varepsilon$ is to increase the temperature profile due to the assumption of temperature dependent thermal conductivity, which makes reduction in the magnitude of the transverse velocity by a quantity $\%$ oy $K(T)$. Also for each value of $\varepsilon$, the thermal boundary layer thickness reduces for unsteady state comparing with steady one. 
Temperature profile for the selected values of space and temperature-dependent heat source/sink parameters are predicted in Fig.10 and Fig.11. It is evident from these figures, the temperature in the thermal boundary layer increases with increase in $A^{*}, B^{*}$ for a given value of $B^{*}, A^{*}$ in both cases of UP and VP. The heat sink $\left(A^{*}, B^{*}<0\right)$ parameters lead to decrease in the thermal boundary layer whereas the boundary layer thickness increases with increase in $\left(\boldsymbol{A}^{*}, \boldsymbol{B}^{*}>0\right)$. Moreover, it is observed that the variation between UP and VP is more for space dependent heat sink parameter $A^{*}<\mathrm{O}$ than heat source parameter $A^{*}>0$, whereas reverse trend is observed in $\boldsymbol{B}^{*}$.

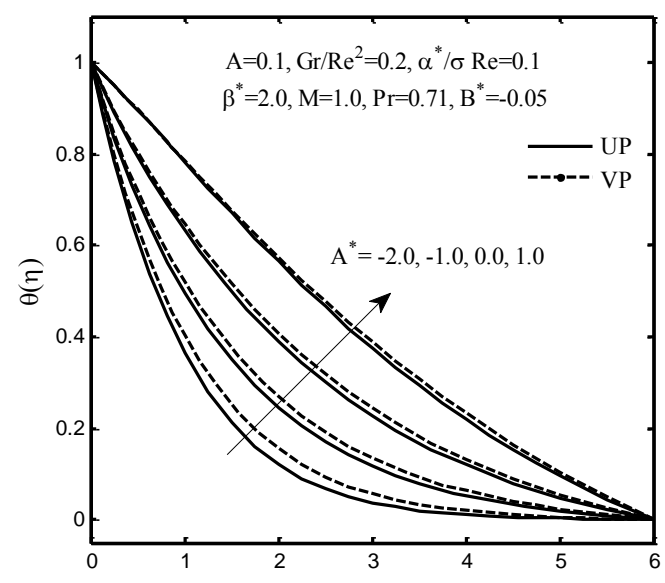

Fig. 10 Temperature profile for different values of space dependent heat source/sink parameters

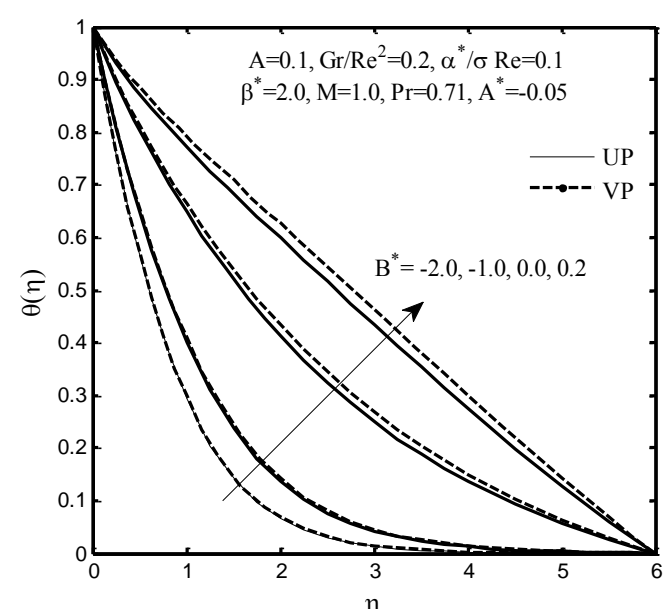

Fig. 11 Temperature profile for different values of temperature dependent heat source/sink parameters

The variation of temperature distribution within the boundary layer for various values of $P r, A$ in both cases of UP and VP are depicted in Fig.12. As $P r$ increases, the temperature is decreasing at a steeper rate in the flow region, which shows that the rate of cooling is much faster and the thermal boundary layer thickness becomes thinner for higher values of
Pr. In the case of steady state and low Prandtl number, the temperature distribution for VP is slightly increasing behavior than in UP. But, there is no such a significant behavior between UP and VP with increase in $\operatorname{Pr}$ and $A$.

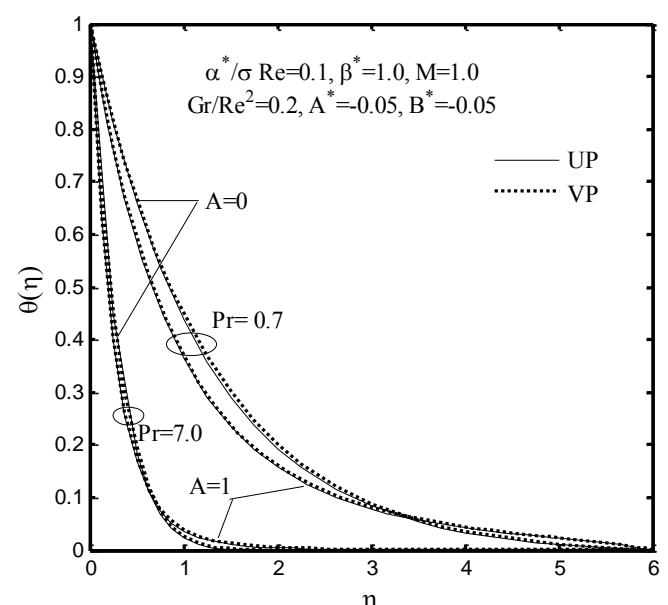

Fig. 12 Temperature profile for different values of Prandtl number and unsteady parameter

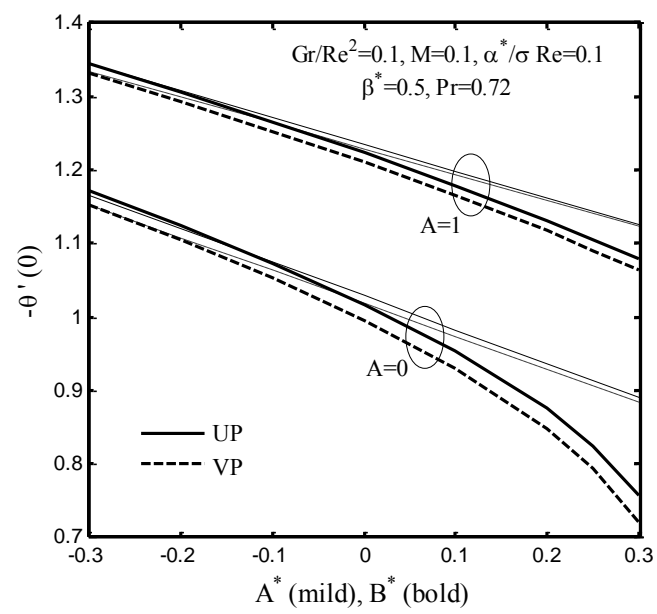

Fig. 13 Variation of Nusselt number with unsteady, non-uniform heat generation parameters

The variation of temperature gradient with unsteady parameter $A$, non-uniform heat generation parameters $\boldsymbol{A}^{*}, \boldsymbol{B}^{*}$ is plotted in Fig.13. It is pointed out that the rate of heat transfer increases with an increase in unsteady parameter and decreases with an increase in non-uniform heat generation parameters $A^{*}, B^{*}$. Also, the heat transfer rate is low in VP comparing with UP with an increase of heat generation parameters. Moreover, the heat transfer rate for time-dependent heat generation parameter $\boldsymbol{B}^{*}>\mathbf{O}$ decreases very fastly than in space dependent heat generation parameter $A^{*}>0$. The positive value of heat transfer rate shows that the heat is transferring from plate to the fluid i.e. cooling of the plate occurs. Thus it can be concluded that the heat sink parameters $\left(A^{*}, B^{*}<0\right)$ can be effectively used for the fast cooling of the plate, as expected. It is 
pointed out that the unsteady term makes to cool the plate faster.

The impact of physical parameters $\lambda, M, \frac{\alpha^{*}}{\sigma \mathrm{Re}}, \beta^{*}, \varepsilon$ on the skin friction co-efficient $f^{\prime \prime}(0)$ and the wall temperature gradient $-\theta^{\prime}(0)$ is given in Table 2. The skin friction co-efficient is governed by the slope of the fluid velocity at the wall. As the fluid velocity increases, the slope of the velocity profile also increases, which tends to increase the skin friction co-efficient. Thus, skin friction increases with increase in $\varepsilon, \lambda$, whereas decreases with increase in $\beta^{*}, \alpha^{*} / \sigma$ Re, $\boldsymbol{M}$. Also, the rate of heat transfer enhances with $\mathrm{A}, \lambda$, but it decreases with increase in $\varepsilon, \beta^{*}, \alpha^{*} / \sigma \operatorname{Re}, M$. It is noted that from the values of UP, VP for each parameter, VP has more tendency to control the fluid velocity than UP. Thus, the study of variable permeability (VP) is more important in fluid flow through porous media.

Table 2 Skin friction and wall temperature gradient for different values of pertinent parameters

\begin{tabular}{|c|c|c|c|c|c|c|c|c|c|}
\hline & & & & & \multirow{2}{*}{\multicolumn{3}{|c|}{$\frac{\left(\mathrm{Pr}=0.72, A^{*}, B^{*}=-0.05\right.}{\mathrm{UP}}$}} & \multirow{2}{*}{\multicolumn{2}{|c|}{ VP }} \\
\hline \multirow[b]{2}{*}{ A } & \multirow[b]{2}{*}{$\lambda=\frac{G r}{\operatorname{Re}^{2}}$} & \multirow[b]{2}{*}{$M$} & \multirow[b]{2}{*}{$\frac{\alpha^{*}}{\sigma \operatorname{Re}}$} & \multirow[b]{2}{*}{$\beta^{*}$} & & & & & \\
\hline & & & & & $\varepsilon$ & $f^{\prime \prime}(0)$ & $-\theta^{\prime}(0)$ & $f^{\prime \prime}(0)$ & $-\theta^{\prime}(0)$ \\
\hline \multirow[t]{2}{*}{0} & $\overline{0.1}$ & "0.1 & 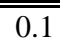 & 0.5 & "0.1 & -1.182891 & 0.796237 & -1.372596 & 0.781352 \\
\hline & & & & & 0.5 & -1.178497 & 0.635081 & -1.368657 & 0.622924 \\
\hline \multirow[t]{2}{*}{1} & 0.1 & 0.1 & 0.1 & 0.5 & 0.1 & -1.470428 & 1.063873 & -1.637654 & 1.055938 \\
\hline & & & & & 0.5 & -1.467828 & 0.862374 & -1.635273 & 0.855915 \\
\hline \multirow[t]{2}{*}{0} & 0.1 & 0.1 & 0.1 & 1.0 & 0.1 & -1.311183 & 0.780905 & -1.664145 & 0.753913 \\
\hline & & & & 2.0 & & -1.538455 & 0.754640 & -2.149738 & 0.711683 \\
\hline \multirow[t]{2}{*}{1} & 0.1 & 0.1 & 0.1 & 1.0 & 0.1 & -1.578446 & 1.056766 & -1.895666 & 1.042267 \\
\hline & & & & 2.0 & & -1.775346 & 1.044597 & -2.338814 & 1.021148 \\
\hline \multirow[t]{2}{*}{0} & 0.1 & 0.1 & 0.5 & 0.5 & 0.1 & -1.338582 & 0.763102 & -1.469628 & 0.757295 \\
\hline & & & 1.0 & & & -1.511511 & 0.728565 & -1.580282 & 0.731771 \\
\hline \multirow[t]{2}{*}{1} & 0.1 & 0.1 & 0.5 & 0.5 & 0.1 & -1.599271 & 1.051072 & -1.717523 & 1.047347 \\
\hline & & & 1.0 & & & -1.747268 & 1.037452 & -1.811530 & 1.037902 \\
\hline \multirow[t]{2}{*}{0} & 0.1 & 0.5 & 0.1 & 0.5 & 0.1 & -1.338582 & 0.763102 & -1.514629 & 0.749923 \\
\hline & & 1.0 & & & & -1.511511 & 0.728565 & -1.674176 & 0.717152 \\
\hline \multirow[t]{2}{*}{1} & 0.1 & 0.5 & 0.1 & 0.5 & \begin{tabular}{|l|}
0.1 \\
\end{tabular} & -1.599271 & 1.051072 & -1.757029 & 1.044068 \\
\hline & & 1.0 & & & & -1.747268 & 1.037452 & -1.895335 & 1.031365 \\
\hline \multirow[t]{2}{*}{0} & 0.5 & 0.1 & 0.1 & 0.5 & 0.1 & -0.998171 & 0.838582 & -1.199267 & 0.823686 \\
\hline & 1.0 & & & & & -0.788033 & 0.875244 & -1.002071 & 0.859801 \\
\hline \multirow[t]{2}{*}{1} & 0.5 & 0.1 & 0.1 & 0.5 & 0.1 & -1.320863 & 1.080417 & -1.495977 & 1.071941 \\
\hline & 1.0 & & & & & -1.140954 & 1.098823 & -1.325673 & 1.089693 \\
\hline
\end{tabular}

\section{CONCLUSION}

In this paper, we have investigated numerically the effect of non-uniform heat generation on unsteady MHD boundary layer flow of an incompressible viscous fluid and heat transfer over a vertical surface embedded in the porous medium with variable porosity and variable thermal conductivity. From the present investigation the following conclusions may be drawn:

1. An increase in the unsteady parameter is to decrease the thickness of the momentum and thermal boundary layers for all the governing parameters.
2. The velocity profile decreases with an increase in the values of magnetic parameter $M$, inertial parameter and the porous parameter, whereas reverse trend is seen with increasing the buoyancy parameter $\lambda$. Also, the temperature profile increases with an increase in the values of magnetic parameter, inertial parameter, variable thermal conductivity parameter, nonuniform heat source parameters whereas it decreases with an increase in buoyancy parameter, Prandtl number, non-uniform heat sink parameters.

3. The value of the local skin-friction coefficient increases with increase in buoyancy parameter whereas reverse effect is seen by increasing 
magnetic parameter, the inertial parameter and the porous parameter. Moreover, the rate of heat transfer decreases with an increase in magnetic, thermal conductivity, inertial, porous and nonuniform heat source parameters.

4. The rate of heat transfer increases with an increase in the unsteady parameter $A$, the buoyancy parameter, non-uniform heat sink parameters $A^{*}, B^{*}<0$. Thus fast cooling of the plate can be achieved by implementing these effects.

5. Variable permeability has more tendency to control the fluid velocity than by applying uniform permeability in the applications.

\section{ACKNOWLEDGEMENTS}

This work was supported by the National Research Foundation Grant funded by the Korean Government (No. 2008-0060153).

\section{REFERENCES}

Al-Nimr, M.A. and S. Masoud (1998) Unsteady free convection flow over a vertical flat plate immersed in a porous medium. Fluid Dyn. Res. 23, 153-160.

Abo-Eldahab, E.M. and M.A. El-Aziz (2004) Blowing/suction on hydromagnetic heat transfer by mixed convection from an inclined continuously stretching surface with internal heat generation/absorption, Int. J. Thermal Sci. 43, 709-719.

Abel, M.S., P.G. Siddheshwar, and M.M. Nandeppanavar (2007) Heat transfer in a viscoelastic boundary layer flow over a stretching sheet with viscous dissipation and non-uniform heat source, Int. J. Heat Mass Transfer. 50, 960-966.

Anand Rao, J., S. Sivaiah and R. Srinivasa Raju (2012) Chemical Reaction Effects on an Unsteady MHD Free Convection Fluid Flow past a Semi-Infinite Vertical Plate Embedded in a Porous Medium with Heat Absorption. Journal of Applied Fluid Mechanics, 5(3), 6370.

Bataller, R.C. (2007) Viscoelastic fluid flow and heat transfer over a stretching sheet under the effects of a non-uniform heat source, viscous dissipation and thermal radiation, Int. J. Heat Mass Transfer. 50, 3152-3162.

Chandrasekhara, B.C. and P.M.S. Namboodiri (1985) Influence of variable permeability on combined vertical surfaces in porous medium. Int. J. Heat Mass Transfer. 28, 199-206.

Chiam, T.C. (1996) Heat transfer with variable conductivity in a stagnation point flow towards a stretching sheet, Int. Commun. Heat Mass Transf. 23, 239-248.
Chiam, T.C. (1998) Heat transfer in a fluid with variable thermal conductivity over a linearly stretching sheet, Acta Mech. 129, 63-72.

Chamkha, A.J. and S.E. Ahmed (2011), Similarity solution for unsteady MHD flow near a stagnation point of a three dimensional porous body with heat and mass transfer, heat generation/ absorption and chemical reaction, Journal of Applied Fluid Mechanics, 4(2), 8794.

Das, K. (2012) Impact of thermal radiation on MHD slip flow over a flat plate with variable fluid properties. Heat Mass Transfer, 48, 767778 .

Ingham, D.B. and I. Pop (1998) Transport phenomena in porous media. Pergamon, Oxford.

Ishak, A., R. Nazar and I. Pop (2009a) Heat transfer over an unsteady stretching permeable surface with prescribed wall temperature, Nonlin. Anal.:Real World Appls. 10, 29092913.

Ishak, A., R. Nazar and I. Pop (2009b) Boundary layer flow and heat transfer over an unsteady stretching vertical surface, Meccanica 44, 369375.

Johnson, C.H. and P. Cheng (1978) Possible similarity solutions for free convection boundary layer adjacent to flat plate in porous media. Int. J. Heat Mass Transfer, 21 709-718.

Lai, F.C. and F.A. Kulacki (1990) The influence of surface mass flux on mixed convection over horizontal plates in saturated porous media. Int. J. Heat Mass Transfer, 33, 576-579.

Mahmoud, M.A.A. (2007) Thermal radiation effects on MHD flow of a micro polar fluid over a stretching surface with variable thermal conductivity, Physica A. 375, 401-410.

Makinde, O.D. and A. Aziz (2010) MHD mixed convection from a vertical plate embedded in a porous medium with a convective boundary condition. Int. J. Thermal Sciences, 49, 18131820.

Nield, D.A. and A. Bejan (1992) Convection in porous media. Springer, New York .

Mahanti, N.C. and P. Gaur (2009), Effects of varying viscosity and thermal conductivity on steady free convective flow and heat transfer along an isothermal vertical plate in the presence of heat sink, Journal of Applied Fluid Mechanics, 2(1), 23-28.

Pantokratoras, A. (2009) A common error made in investigation of boundary layer flows, Applied Mathematical Modelling 33, 413-422. 
Pal, D. (2011) Combined effects of non-uniform heat source/sink and thermal radiation on heat transfer over an unsteady stretching permeable surface, Commun. Nonlinear Sci. Numer. Simulat. 16, 1890-1904.

Pal, D. and H. Mondal (2011) The influence of thermal radiation on hydromagnetic DarcyForchheimer mixed convection flow past a stretching sheet embedded in a porous medium. Meccanica, 46, 739-753.

Prasad, K.V., K. Vajravelu and A. Sujatha (2013) Influence of internal heat generation/absorption, thermal Radiation, magnetic field, variable fluid property and viscous dissipation on heat transfer characteristics of a Maxwell fluid over a stretching sheet, Journal of Applied Fluid Mechanics, 6(2), 249-256.

Prakash, D., M. Muthtamilselvan and D.H. Doh (2012) Effect of heat generation on forced convection through a porous saturated duct. Transp. Porous Med., 95, 377-388.

Prakash, D., M. Muthtamilselvan and D.H. Doh (2014) Unsteady MHD non-Darcian flow over a vertical stretching plate embedded in a porous medium with non-uniform heat generation. Appl. Math. Comput., 236, 480492.

Riley, N. (1964) Magnetohydrodynamic free convection, J. Fluid Mech. 18, 577-586.

Raptis, A.A. (1983) Unsteady free convection flow through a porous medium. Int. J. Eng. Sci. 21, 345-348.

Rahman, M.M., M.J. Uddin and A. Aziz (2009) Effects of variable electric conductivity and non-uniform heat source (or sink) on convective micro polar fluid flow along an inclined flat plate with surface, Int. J. Thermal Sci. 48, 23312340.

Reddy, T.S., M. C. Raju and S. V. K. Varma (2013) Unsteady MHD radiative and chemically reactive free convection flow near a moving vertical plate in porous medium. Journal of Applied Fluid Mechanics, 6(3), 443-451.

Sakiadis, B.C. (1961) Boundary layer behavior on continuous solid surfaces. AIChE J. 7, 26-28.

Sparrow, M.E. and R.D. Cess (1961) Effect of magnetic field on free convection heat transfer. Int. J. Heat Mass Transfer. 3, 267-274.

Sing, K.R. and T.G. Cowling (1963) Thermal conduction in magnetohydrodynamics. $J$. Mech. Appl. Math. 16, 1-5.

Savvas, T.A., N.C. Markatos and C.D. Papaspyrides (1994) On the flow of nonNewtonian polymer solutions, Appl. Math. Modeling. 18, 14-21.

Sharma, P.R. and G. Singh (2009), Effects of variable thermal conductivity and heat source/sink on MHD flow near a stagnation point on a linearly stretching sheet, Journal of Applied Fluid Mechanics, 2(1), 13-21.

Vafai, K. and C.L. Tien (1981) Boundary and inertia effects on flow and heat transfer in porous media. Int. J. Heat Mass Transfer, 24, 195-203.

Vajravelu, K. and K.V. Prasad and Chiu-On Ng (2013) Unsteady convective boundary layer flow of a viscous fluid at a vertical surface with variable fluid properties, Nonlin. Anal.:Real World Appls. 14, 455-464. 